\title{
Short Pulse Laser Absorption and Energy Partition at Relativistic Laser Intensities
}

R. Shepherd, H. Chen, Y. Ping, G. Dyer, S. Wilks, H.-K. Chung, A. Kemp, S. Hanson, K. Widmann, K. Fournier, A. Faenov, T. Pikuz, A. Niles, P. Beiersdorfer

March 30, 2007 
This document was prepared as an account of work sponsored by an agency of the United States Government. Neither the United States Government nor the University of California nor any of their employees, makes any warranty, express or implied, or assumes any legal liability or responsibility for the accuracy, completeness, or usefulness of any information, apparatus, product, or process disclosed, or represents that its use would not infringe privately owned rights. Reference herein to any specific commercial product, process, or service by trade name, trademark, manufacturer, or otherwise, does not necessarily constitute or imply its endorsement, recommendation, or favoring by the United States Government or the University of California. The views and opinions of authors expressed herein do not necessarily state or reflect those of the United States Government or the University of California, and shall not be used for advertising or product endorsement purposes.

This work was performed under the auspices of the U.S. Department of Energy by University of California, Lawrence Livermore National Laboratory under Contract W-7405-Eng-48. 


\title{
FY06 LDRD Final Report Short Pulse Laser Absorption and Energy Partition at Relativistic Laser Intensities LDRD Project Tracking Code: 04-ERD-023 Ronnie Shepherd, Principal Investigator
}

\begin{abstract}
We have performed experiments at the COMET and Calisto short pulse laser facilities to make the first comprehensive measurements of the laser absorption and energy partition in solid targets heated with an ultrashort laser pulse focused to relativistic laser intensities $\left(>10^{17}\right.$ $\left.W / \mathrm{cm}^{2}\right)$. The measurements show an exceedingly high absorption for $P$ polarized laser-target interactions above $10^{19} \mathrm{~W} / \mathrm{cm}^{2}$. Additionally, the hot electron population is observed to markedly increase at the same intensity range. An investigation of the relaxation process was initiated using time-resolved $K_{\alpha}$ spectroscopy. Measurements of the time-resolved $K_{\alpha}$ radiation suggest a 10-20 ps relativistic electron relaxation time. However modeling difficulties of these data are apparent and a more detailed investigation on this subject matter is warranted.
\end{abstract}

\section{Introduction/Background}

Currently ultrashort pulse lasers are used to heat matter at high intensities $\left(>10^{17} \mathrm{~W} / \mathrm{cm}^{2}\right)$ at laboratories throughout the world ${ }^{1,2,3}$. The experiments being done at these intensities range from fast ignitor research for inertial confinement fusion (ICF) ${ }^{4}$ to short pulse $\mathrm{x}$-ray generation ${ }^{5}$. At these intensities, physical effects that are small at low intensities become dominant. The pondermotive force begins to alter the density profile, an electrostatic driven ion-shock is predicted to form, the relativistic mass of the electrons increase the penetration depth of the laser, and magnetic fields that can exceed 3 gigaGauss are generated inside the target. Furthermore, the oscillation velocity $\left(\mathrm{v}_{\mathrm{os}}\right)$ becomes large compared to thermal velocity $\left(\mathrm{v}_{\mathrm{th}}\right)$, suggesting a significant amount of vacuum heating and relativistic $\mathrm{JxB}$ heating. Although high intensity laser matter applications would benefit greatly from understanding the interaction physics, little experimental work has been done to study the details of the laser light absorption and subsequent energy partition at these high intensities leaving a somewhat inadequate data set in the regime. Similarly, when considering a model for high intensity, laser-matter interactions the models are equally inadequate at describing the interaction physics. The absorption is typically modeled using one of three methods; 1) A particle-in-cell (PIC) code, 2) by solving the Helmholtz wave (for fluid codes such as LASNEX), or 3) an absorbed energy is assumed before expansion occurs. All these techniques make approximations that result in substantial inaccuracies in modeling the physics of the interaction process. The PIC codes ignore collisions, making it difficult to compare calculations with one of the most useful plasma diagnostics (x-ray spectroscopy), the Helmholtz wave equation fails to consider the long particle mean-free-paths as compared 
to the cell size, and the assumption of energy deposition before expansion depends on knowledge of the fractional absorption while neglecting energy loss due to hydrodynamic motion. Ultimately, all high intensity laser-matter experiments would benefit greatly from a detailed study of the absorption process and subsequent partitioning of energy (radiation, hydrodynamic motion, energetic particles, and bulk thermal heating).

In our research, we perform two experiments that provide important information about laser absorption at relativistic laser intensities and the relaxation of relativistic electrons in high-density matter. By pushing the limits of diagnostics and increasing the knowledge base through careful experiment, we have developed a better understanding of the underlying physics involved in the energy partition. This knowledge is essential for designing and interpreting current and future HED experiments.

\section{Research Activities}

The first experiment looks at the absorption of laser light when focused to relativistic intensities on solid targets. The energy of laser pulses is coupled into targets through various mechanisms. In the relativistic regime, where the peak oscillatory velocity of electrons in the laser field, $\mathrm{v}_{\text {osc }}$, is an appreciable fraction of the speed of light c (e.g. $\mathrm{v}_{\text {osc }} / \mathrm{c} \sim 0.2$ at $10^{17} \mathrm{~W} / \mathrm{cm}^{2}$, and $\mathrm{v}_{\text {osc }} / \mathrm{c} \sim 0.99$ at $10^{20} \mathrm{~W} / \mathrm{cm}^{2}$ ), there are three major absorption processes ${ }^{6}$ : resonant absorption, $\mathrm{J} \times \mathrm{B}$ heating and vacuum heating. Resonant absorption occurs when an obliquely incident laser pulse interacts with a solid target where low-density plasma formed by prepulses exists at the surface (preplasma). A plasma wave can be resonantly driven by the laser field, creating hot electrons at the front side of the target. The $\mathrm{J} \times \mathrm{B}$ heating is due to the ponderomotive force of the laser pulse that is generated by the gradient of the intense light pressure. As a result, the hot electrons produced by $\mathrm{J} \times \mathrm{B}$ heating usually follow the laser propagation direction ${ }^{7}$. Vacuum heating occurs when a laser pulse is incident onto the target at an oblique angle. The component of the laser electric field perpendicular to the target surface accelerates electrons nonadiabatically if the interface is sharp enough, leading to hot electrons emitted in a direction normal to the target $^{8}$. Since different mechanisms lead to hot electrons with different spatial features, as illustrated in Fig.1, the absorption physics can be revealed by measuring the spatial distribution of hot electrons.

The laser absorption experiment was performed on the LLNL Callisto laser facility at Lawrence Livermore National Laboratory (LLNL). Callisto is a Ti:sapphire laser system delivering 150-fs laser pulses at $800 \mathrm{~nm}$ with energies up to $20 \mathrm{~J}$. The p-polarized laser pulse was focused by an $\mathrm{f} / 3$ off-axis parabola onto the target at an incident angle of either $6^{\circ}$ or $45^{\circ}$. The focal spot at normal incidence contained $50 \%$ of the energy within $5-\mu \mathrm{m}$ full width at half maximum (FWHM), resulting in a maximum intensity of $3 \times 10^{20}$ $\mathrm{W} / \mathrm{cm}^{2}$. Side-on interferometric measurements show that the scale length $\mathrm{L}(\mathrm{L} \equiv$ $\left(\partial \ln \left(n_{e}\right) / \partial z\right)^{-1}$ where $n_{e}$ is the plasma electron density) of the preplasma was randomly distributed in the range of $3-10 \mu \mathrm{m}(3 \mu \mathrm{m}$ is the resolution limit of the optical interferometer). 
Absorption. A custom-designed 30-cm-diameter integrating sphere calibrated at the laser wavelength of $800 \mathrm{~nm}$ was employed to collect the scattered laser light $\left(\mathrm{E}_{\text {scatter }}\right)-$ the same technique used in previous low-intensity studies ${ }^{9,10}$. The inner wall of the sphere was coated by a Lambertian scattering material - Spectralon, which has a reflectance of $>$ $98 \%$ over the spectral range of $300-2000 \mathrm{~nm}$. In our initial experiment ( Fig. 2b), $\mathrm{E}_{\text {scatter }}$ was measured by the sphere photodiode covered by an 800 -nm interference filter with a bandwidth of $20 \mathrm{~nm}$. In a follow-up experiment ( Fig. 2b), a visible spectrometer (200$1100 \mathrm{~nm}$ ) was mounted onto the sphere and energy-calibrated at $800 \mathrm{~nm}$ to measure $\mathrm{E}_{\text {scatter }}$. This visible spectrometer and another near-infrared spectrometer $(700-2200 \mathrm{~nm})$ were also used to measure space-integrated energy fraction in harmonics $(2 \omega, 3 / 2 \omega$, and $1 / 2 \omega)$ The sphere had open ports for laser incidence and target alignment. The energy components not captured by the sphere, including backscattered energy $\left(\mathrm{E}_{\text {back }}\right.$, collected by a $2 \%$ beam splitter), reflected energy $\left(\mathrm{E}_{\text {reflect }}\right)$, and transmitted energy $\left(\mathrm{E}_{\text {trans }}\right)$, were measured by calibrated pyroelectric calorimeters. The total input energy $\left(\mathrm{E}_{\text {in }}\right)$ was determined by measuring a leak pulse that was cross-calibrated with the main pulse. The energy deposited in the target was then calculated by $E_{a b s}=E_{\text {in }}-E_{\text {back }}-E_{\text {reflect }}-E_{\text {trans }}-$ $\mathrm{E}_{\text {scatter }}$ (the energy fraction in harmonics was found to be negligible). The absorption fraction is simply $E_{\text {abs }} / E_{\text {in }}$. Since the absorption is usually dependent on the scale length of the preplasma, a side-on interferometer with a 100 -fs probe pulse at $400 \mathrm{~nm}$ was set up to monitor the preplasma density profile. The probe was timed $10 \mathrm{ps}$ earlier than the main pulse. An electron spectrometer, located at $240^{\circ}$ as defined in Fig. 3a, was used to sample the energy spectra of escaped hot electrons at the backside.

Dosimetry. The integrating sphere was replaced by a 30 -cm-diameter dosimeter holder located in the laser incident plane with the target at the center. The thermoluminescence dosimeters (TLD) were covered by 100 - or $500-\mu \mathrm{m}$-thick aluminium filters to block electrons with energies below $120 \mathrm{keV}(340 \mathrm{keV}$ for $500-\mu \mathrm{m}$ filters), x-rays with energies below $9 \mathrm{keV}$ ( $15 \mathrm{keV}$ for $500-\mu \mathrm{m}$ filters), and most ions. The ultrathin thickness of TLD ensures that the hot electron contribution is dominant over the $\mathrm{x}$-ray contribution to the dose $\mathrm{s}^{11,12}$. The measured energy absorption as a function of laser intensity for near-normal incidence $\left(6^{\circ}\right)$ and oblique incidence $\left(45^{\circ}\right)$ is plotted in Fig. $2 \mathrm{a}$ and $2 \mathrm{~b}$, respectively. Previously published $\operatorname{data}^{13}$ for normal incidence at low intensities are also plotted in Fig. 2a for comparison. Because the previous measurements were preformed at a different laser wavelength $(\lambda=400 \mathrm{~nm})$, the horizontal coordinate of Fig. $2 \mathrm{a}$ is $\mathrm{I} \lambda^{2}$, which is proportional to the square of the electron quiver momentum in the laser field. Our measurements are consistent with previously published data at low intensities $\left(\mathrm{I} \sim 10^{17} \mathrm{~W} / \mathrm{cm}^{2}\right)$, corroborating the validity of our measuring method. As the intensity increases, the absorption rises up to more than $60 \%$ at $\mathrm{I} \sim 10^{20} \mathrm{~W} / \mathrm{cm}^{2}$. For $45^{\circ}$ incidence, two sets of data were taken with slightly different methods. As shown in Fig. $2 \mathrm{~b}$, they are in good agreement with each other. The absorption hovers around 50\% from $10^{17} \mathrm{~W} / \mathrm{cm}^{2}$ to $\sim 10^{19} \mathrm{~W} / \mathrm{cm}^{2}$, and then increases with the intensity, reaching a surprisingly high value (80-90\%) at $\mathrm{I}>10^{20} \mathrm{~W} / \mathrm{cm}^{2}$. The relatively large fluctuations in the lowintensity data are due to the variation of scale length from shot to shot. However, as the intensity reaches above $10^{20} \mathrm{~W} / \mathrm{cm}^{2}$, the fluctuation is significantly smaller, showing that the absorption becomes less dependent on the scale length, as discussed later with the identification of the mechanisms. The simulation results, shown in both plots, were 
obtained from two-dimensional particle-in-cell (2D PIC) calculations using ZOHAR ${ }^{14}$, with a 6 - $\mu \mathrm{m}$-scale-length preplasma and an initial electron density of $7 \times 10^{22} \mathrm{~cm}^{-3}$ (an order of magnitude less than the solid density). The calculated absorption agrees well with the measured values in the range of $10^{18}-10^{19} \mathrm{~W} / \mathrm{cm}^{2}$, but under estimates the absorption as the intensity approaches $10^{20} \mathrm{~W} / \mathrm{cm}^{2}$. This is probably due to the maximum density imposed by computational limitations and lack of transport processes in the code. The measurements presented here certainly provide a benchmark for future improvement of simulation tools in the ultra-relativistic regime.

The spatial features of hot electrons provide a signature of the dominant absorption mechanism. The measured angular distributions of hot electrons by dosimeters with a resolution of $10^{\circ}$ at three intensities are shown in Fig. 3. At a low intensity of $8 \times 10^{17}$ $\mathrm{W} / \mathrm{cm}^{2}$ (top), most hot electrons are emitted at the front side as shown in the measurement, consistent with the resonant absorption mechanism. At an intermediate intensity of $2 \times 10^{19} \mathrm{~W} / \mathrm{cm}^{2}$ (middle), the backside component increases to become comparable with the front-side part. There is a distinct peak in the laser direction, showing that the $\mathrm{J} \times \mathrm{B}$ heating starts to play a role. As the intensity approaches $2 \times 10^{20}$ $\mathrm{W} / \mathrm{cm}^{2}$ (bottom), the measurement shows that hot electrons at the backside are dominant and the maximum dose is located at the target normal direction, indicating that vacuum heating has become the most important absorption mechanism. The energy spectra of hot electrons, measured simultaneously with the dosimetry at $240^{\circ}$ at the backside of the target, show that the temperature of the hot electrons reaches $200 \mathrm{keV}$ at $\mathrm{I}=2 \times 10^{19}$ $\mathrm{W} / \mathrm{cm}^{2}$, and $1.2 \mathrm{MeV}$ at $\mathrm{I}=2 \times 10^{20} \mathrm{~W} / \mathrm{cm}^{2}$.

We have identified changes in the dominant absorption mechanism as a function of the laser intensity for the case of oblique incidence. Since there is always some preplasma in front of the target surface as commonly observed in most high-energy laser systems, resonant absorption always contributes to the laser energy deposition at oblique incidence. The $\mathrm{J} \times \mathrm{B}$ heating does not play a major role until the intensity reaches $10^{19}$ $\mathrm{W} / \mathrm{cm}^{2}$ simply because the ponderomotive force is not strong enough at lower intensities. The criterion for effective vacuum heating is $\mathrm{x}_{\mathrm{osc}}=\mathrm{v}_{\mathrm{osc}} / \omega>\mathrm{L}$, where $\mathrm{x}_{\mathrm{osc}}$ is the oscillatory amplitude of electrons in the laser field and $\omega$ is the laser frequency ${ }^{15}$. At weakly relativistic intensities $\left(\sim 10^{17} \mathrm{~W} / \mathrm{cm}^{2}\right), \mathrm{x}_{\text {osc }} \sim 0.03 \mu \mathrm{m}$, while at $10^{20} \mathrm{~W} / \mathrm{cm}^{2}, \mathrm{x}_{\text {osc }}$ increases to $\sim 1 \mu \mathrm{m}$, which is closer to the scale length $(3-10 \mu \mathrm{m})$ measured 10 ps before the arrival of the main pulse. The fact that the preplasma density profile is steepened by the ponderomotive force during the laser pulse ${ }^{16,17}$ makes it possible for the vacuum heating to play a major role at the highest intensities. This is also consistent with the enhanced absorption as the intensity rises above $10^{20} \mathrm{~W} / \mathrm{cm}^{2}$ (Fig. 2b) since an additional absorption mechanism, vacuum heating, comes into play.

A rough estimate of the total energy in the hot electrons based on dosimetric measurements shows that the escaped hot electrons account for less than $1 \%$ of the total laser energy. Most hot electrons are actually trapped inside the target by the Coulomb barrier and transfer their energy to the background electrons, ultimately heating ions in the solid ${ }^{1825}$. The measured high absorption $\left(80-90 \%\right.$ for $45^{\circ}$ incidence at $\mathrm{I}>10^{20} \mathrm{~W} / \mathrm{cm}^{2}$ ) 
indicates an efficient coupling of the laser energy into the target, which, if properly channeled by target design, could potentially improve the efficiency in applications such as the generation of monoenergetic ions ${ }^{19,20}$ and fast ignition. The observed characteristics in the spatial distributions of hot electrons will also help guide experimental studies. For example, a detector located in a direction normal to the target will offer the best chance to observe electron-positron pairs, since vacuum heating is dominant at ultrahigh intensities. Furthermore, the directional hot electrons emitted from the target could be utilized to simulate the high-energy particle jets from astrophysical objects in a laboratory ${ }^{21}$. The present study provides unprecedented insight into the absorption physics of ultra-intense laser pulses, and we expect that our results will have broad impact on both basic research and applications in this inspiring ultra-relativistic regime.

The second experiment looks at the dissipation of energy by the relativistic electrons. The interaction of high-intensity lasers with solid targets generates relativistic electrons in laboratory plasmas. These electrons, which are accelerated by the interaction with the laser electric field or the associated ponderomotive force, can effectively heat the solid target beyond the region of direct laser interaction. One important application of relativistic electron isochoric heating of solid targets is fast ignition. To this goal, substantial effort has been made in past decades to understand the conversion efficiency of intense laser light to fast electrons and their transport through the target.

A well-established technique is to infer the absolute number of the laser-produced electrons by detecting the electron-induced $\mathrm{K}_{\alpha}$ fluoresence emission from buried layer targets. The copious amounts of hot electrons are produced in the interaction of intense laser pulses with solid targets ${ }^{22}$. What is not so well known is how these hot electrons couple to the rest of the solid target. It is important since applications rely on the rapid coupling of the hot electrons to the background electrons before the targets disassemble hydro-dynamically. In fast ignition, for example, this time is currently thought to be less than 20 ps.

$\mathrm{K}_{\alpha}$ radiation is generated when energetic electrons excite the K-shell electrons of atoms; it is commonly used to estimate the total number of hot electrons generated during laser-matter interaction ${ }^{23,24,25,26,27}$, and, by using layered targets of different materials, to determine their spatial distribution in the target ${ }^{4,28}$. Previous $\mathrm{K}_{\alpha}$ measurements have all been time-integrated, implying that the radiation is generated instantaneously. We present the first picosecond time-resolved measurements of the relaxation of hot electrons generated by a short pulse, high intensity laser. Our measurements indicate that most of the $\mathrm{K}_{\alpha}$ radiation is generated long $(10-20 \mathrm{ps})$ after the laser interaction. This suggests that the electrons responsible for the underlying atomic processes are not laser-generated hot electrons, but rather electrons that have originated in an ionization cascade initiated by the hot electrons. Hence, ionization cascade should not be ignored, contrary to the assumption of many previous measurements, when hot-electron population is deduced from $\mathrm{K}_{\alpha}$ emission measurement. The data is interpreted using a simple model based on collisional coupling, plasma expansion and M-shell ionization that can reproduce basic characteristics of the measured $\mathrm{K}_{\alpha}$ history. Our findings shed new light on the current interpretation of a major diagnostic tool for many experiments in short-pulse laser matter interaction. 
The experiment was performed at the Compact Multipulse Terawatt (COMET) laser facility ${ }^{29}$ at Lawrence Livermore National Laboratory. COMET is a hybrid chirped pulse amplification (CPA) system with a Ti:sapphire oscillator and regenerative amplifier with a four-stage Nd:phosphate glass amplifier. The laser wavelength is $1054 \sim \mathrm{nm}$. For our data, the laser pulse length was 500 fs at full width at half maximum (FWHM) and p-polarized. The laser energy was $6-7 \mathrm{~J}$ focused with an $\mathrm{f} / 3.6$ parabola on solid targets at an incident angle of $45^{\circ}$. The best focus was typically 8-10 $\mu \mathrm{m}$ FWHM, resulting in a laser intensity of $10^{19} \mathrm{Wcm}^{2}$ at the best focus. By systematically increasing the spot size on the target, we were able to decrease the laser intensity down to $10^{17} \mathrm{Wcm}^{2}$ while keeping the laser energy constant. Pre-pulse is a major concern in short-pulse, laser-solid experiments. The temporal intensity contrast of the pulses is characterized by three components: 1) a 12 ns pre-pulse produced by leakage in the regenerative amplifier, 2) amplified spontaneous emission (ASE), and 3) a pedestal produced by the third and fourth order abberations in the stretcher. Often the most significant pre-pulse comes from the leakage pulse from the regenerative amplifier. Using two Pockels cells and polarizers, a contrast of $\mathrm{I}_{\text {leakage }} / \mathrm{I}_{\text {main }} \sim 10^{-9}$ was obtained. No measurement was made of the contribution of ASE or third and fourth order aberrations. However, an analysis of the spectrum from the stretcher-compressor system suggest the contribution of the third and fourth order aberrations to non-Gaussian components produce a contrast of $\mathrm{I}_{\text {aberr }} / \mathrm{I}_{\text {main }} \sim 10^{-5}$ at about 2 ps before the peak of the pulse ${ }^{30}$. To confirm that the ASE level produced no pre-formed plasma, solid targets were illuminated without seeding the amplifiers with the oscillator pulse to determine the effect the ASE has on the target. No damage was observed, suggesting minimal effect from ASE. As a monitor of the pre-formed plasma, a visible spectrometer was set up to look at the specular reflected laser light. Because Raman scattering and two-plasmon decay are a strong function of density scale length ${ }^{31}$, the spectrally resolved specular light provided a shot-to-shot monitor of large fluctuations in the pre-pulse. We observed constant harmonic spectra throughout the experiments thatindicates stable preplasma conditions during the laser-target interactions.

The diagnostic setup relative to the laser target is illustrated in figure 4. The targets consisted of $12.5 \mu \mathrm{m}$ of Ti over-coated with $1000 \AA$ of Al. The Al layer prevented direct illumination of the Ti foil thus eliminating any direct heating from the laser. The $\mathrm{x}-$ ray emission from the target was collected using the Time REsolved X-ray Streak camera $(\text { TREX) })^{32}$ interfaced to two von Hámos crystals. An $11 \mathrm{~cm}$ radius of curvature graphite crystal was used to collect the $\mathrm{Ti} \mathrm{K}_{\alpha}$ emission while a $3 \mathrm{~cm}$ radius of curvature crystal was used to collect the $\mathrm{Al} \mathrm{K}$-shell $1 \mathrm{~s} 2 \mathrm{p}\left({ }^{1} \mathrm{P}\right)-1 \mathrm{~s}^{2}\left({ }^{1} \mathrm{~S}\right)\left(\mathrm{He}_{\alpha}\right)$ emission. The streak camera was interfaced to an image intensifier, a 2:1 fiber optic reducer, and a fiber optic face plate mounted on a 16-bit, water-cooled, SITe TK 1024 x 1024 pixel CCD. The time resolution of the streak camera is about $1 \mathrm{ps}$, and the total coverage is about $400 \mathrm{ps}$. A time-integrated measurement of the electrons escaping the target was made using a fiberoptic array compact electron spectrometer (FACES) ${ }^{33}$, which was absolutely calibrated and setup to measure electrons from $80 \mathrm{keV}$ to $6 \mathrm{MeV}$. It was positioned $37 \mathrm{~cm}$ behind the target on the mid-plane, $15^{\circ}$ off the laser propagation direction with a solid angle of 1 x $10^{-4}$ steradians. In addition, a time-integrated, spatially resolved x-ray spectrometer using a spherically bent quartz crystal was fielded to view the back surface of the target from which the heating of Ti target was determined ${ }^{34}$. 
The data were collected at laser focal intensities of $10^{17}, 10^{18}$, and $10^{19} \mathrm{~W} / \mathrm{cm}^{2}$ that correspond to, respectively, non-relativistic $\left(\mathrm{P}_{\mathrm{os}} / \mathrm{m}_{\mathrm{e}} \mathrm{c}=0.3\right.$, where $\mathrm{P}_{\mathrm{os}}$ is the electron momentum, $\mathrm{m}_{\mathrm{e}}$ the electron mass), near-relativistic $\left(\mathrm{P}_{\mathrm{os}} / \mathrm{m}_{\mathrm{e}} \mathrm{c}=1\right)$, and relativistic $\left(\mathrm{P}_{\mathrm{os}} / \mathrm{m}_{\mathrm{e}} \mathrm{c}\right.$ =3) laser intensities. As the laser intensity becomes relativistic, significantly more hot electrons are measured by the electron spectrometer. The number of electrons per $\mathrm{keV}$ at around $100 \mathrm{keV}$ is $4 \times 10^{5}, 9 \times 10^{5}$ and $2 \times 10^{6}$ for the three laser intensities, respectively. This increase is consistent with the enhanced laser absorption at higher laser intensities ${ }^{35}$. A quantitative description of the hot electrons is the so-called "hot electron temperature" $\left(\mathrm{T}_{\mathrm{h}}\right)$, obtained by fitting the spectrum with a single or multiple Maxwellian distributions. To estimate the $T_{h}$ right after the laser pulse from these time integrated spectra, we fit a Maxwellian to the high end of the energy distribution, reasoning that the hot electron energy can only decrease after the laser pulse is turned off. Fits to the high energy section of the electron distributions result in $100 \mathrm{keV}, 270 \mathrm{keV}$ and $520 \mathrm{keV}$ temperature for the intensities of $10^{17}, 10^{18}$, and $10^{19} \mathrm{~W} / \mathrm{cm}^{2}$ respectively. These temperatures agree approximately with the pondermotive scaling of hot electrons except for the highest intensity, where the measured $\mathrm{T}_{\mathrm{h}}$ is about half of the predicted value.

The angular dependence of the hot electron distributions from short-pulse solid interactions has been found experimentally and explained theoretically ${ }^{36,37,38,39}$. For our case (a high contrast P-polarized laser incident at $45^{\circ}$ to a foil target), the most dramatic angular variations would be collimated hot electrons jets. At the intensities of $10^{17}$ and $10^{18} \mathrm{~W} / \mathrm{cm}^{2}$ these jets form mostly at the specular and target normal directions at the front of the target ${ }^{40,41}$ driving resonant absorption, and at $10^{19} \mathrm{~W} / \mathrm{cm}^{2}$ another jet would form at the back of the target due to the poderomotive heating. The angle of this jet is predicted to be at about $20^{\circ}$ off laser direction ${ }^{38,41}$. Since the FACES measurement looks at the back of the target at $15^{\circ}$ off the laser direction, it is not in the path of the possible jets. We estimate the FACES measurements to be within $10 \%$ to $35 \%$ of the $2-\pi$ average of any plausible anisotropic distribution.

Shielded by the Al layer and consequently from direct laser heating, the Ti layer interacts with hot electrons, which excite/ionize K-shell electrons and produce $\mathrm{Ti}$ $\mathrm{K}_{\alpha}$ emission. Because of the extremely short radiation life time of the upper $(n=2)$ level (about 5 fs for Titanium $\mathrm{K}_{\alpha 1}{ }^{42}$ ), for the ps time scale we are considering here, the line intensity of the $\mathrm{Ti}_{\alpha}$ is dictated by electron-impact ionization of the $n=1$ level. The intensity is thus:

$$
I_{K \alpha}=A \int_{V, E} n_{i} n_{e}(E) \sigma(E) v_{e} d V d E
$$

where $\mathrm{n}_{\mathrm{i}}$ is the solid density of $\mathrm{T}_{\mathrm{i}}, \mathrm{n}_{\mathrm{e}}(\mathrm{E})$ the electron density at energy $E, \sigma(\mathrm{E})$ the cross section of electron impact ionization at energy $E$ and $v_{\mathrm{e}}$ the velocity of electrons. The integral is over the emission volume $V$ and electron energy $E$. Figure 4 shows the measured the time history of the $\mathrm{Ti}_{\alpha}$ intensities for the three laser intensities. We measure the hot-electron relexation time (defined as the FWHM of $I_{K \alpha}$ ) to be 15.9, 13.2, and $12.3 \mathrm{ps}$, for the laser intensities $10^{19}, 10^{18}$, and $10^{17} \mathrm{~W} / \mathrm{cm}^{2}$, respectively. Note that the $\mathrm{K}_{\alpha}$ emission durations in our experiment are significantly larger than those measured previously $^{43,23}$. This is due to (i) our hot, electron temperatures are at least one order of magnitude larger; (ii) as a consequence, the hot electron range here is many times the 
target thickness so that multiple interaction and $\mathrm{K}_{\alpha}$ generation can occur with parts of the target that are transparent for $\mathrm{K}_{\alpha}$ radiation. This is not the case in the earlier measurements quoted; (iii) we use different materials with a larger threshold energy for $\mathrm{K}_{\alpha}$.

Collisions between the hot electrons and the bulk thermal electrons increase the temperature of the bulk target. An analysis of our spherical crystal spectrometer measurements indicated a final bulk temperature $T_{c} \sim 50 \mathrm{eV}$ and an average $T_{t}$ ion charge of $4-5^{13}$. The Al layer is also partially heated by the hot electrons and remains hot enough to emit significant $\mathrm{Al} \mathrm{He}{ }_{\alpha}$ radiation long after the Ti- $\mathrm{K}_{\alpha}$ emission has decayed. The inset of figure 5 shows the $\mathrm{Al} \mathrm{He}_{\alpha}$ emission over at least 40 ps (the time coverage of thee streak camera). We have not been able to quantify how much of the Al plasma heating is due to the hot-electron relaxation and how much is due to direct interactions with the laser.

We developed an expanding multi-component plasma model to help understand the measured hot-electron relaxation times. In this model, the hot electrons can lose energy in two ways: through adiabatic cooling due to plasma expansions and through direct collisions that transfer energy from the hot electrons to cold electrons and ions.

The expansion of the plasma is determined by the ion sound speed. Since the hot hot electrons move much faster than the ions ( $v_{i}<0.1 c$ for protons typically), $T_{h}$ evolves adiabatically according to $\boldsymbol{p} \propto \boldsymbol{V}^{\gamma}$, where the volume $V$ is defined by the ion-front position. Combining expansion and collisions gives:

$$
\begin{aligned}
\frac{d T_{h}}{d t} & =v_{e}^{h c}\left(T_{c}-T_{h}\right)+\alpha V(t)^{-\gamma} d V(t) / d t, \\
\frac{d T_{c}}{d t} & =v_{e}^{c h}\left(T_{h}-T_{c}\right),
\end{aligned}
$$

where $\boldsymbol{v}_{e}^{h c}$ is an energy transfer rate between hot and cold electrons given by

$$
\boldsymbol{v}_{\boldsymbol{e}}^{h c}=\frac{8 \sqrt{2 \pi} e^{4}}{3} \frac{\boldsymbol{Z}_{h}^{2} \boldsymbol{Z}_{c}^{2} \ln \Lambda}{\left(\boldsymbol{m}_{\boldsymbol{e}}\left(\boldsymbol{k} \boldsymbol{T}_{\boldsymbol{c}}+\boldsymbol{k} \boldsymbol{T}_{\boldsymbol{h}}\right)\right)^{3 / 2}}
$$

Here, $\boldsymbol{\alpha}=\boldsymbol{T}_{\boldsymbol{h} 0} \boldsymbol{V}_{0}^{\gamma-1}$ depends o the initial hot electron temperature $\boldsymbol{T}_{\boldsymbol{h} 0}$ and the initial target volume $V_{0}$, and $\gamma=5 / 3$. The above equations are complemented by an adequate model for the ion-front position ${ }^{44}$. The predictions for this simplified relaxation model under conditions that are roughly comparable to the experiment for a laser intensity of $10^{19}$ $\mathrm{W} / \mathrm{cm}^{2}$ are shown in the inset of figure 5. The model predicts an initial drop in the $T_{h}$ over the first $\sim 5$ ps due to expansion and an equilibration after $\sim 20$ ps due to collisions.

We have used the measured hot electron temperature and the relaxation profile from the above model with the collisional-radiative code $\mathrm{FLYCHK}^{45}$ to derive the Ti $\mathrm{K}_{\alpha}$ profile under the assumption that the $\mathrm{K}_{\alpha}$ signal is due soley to laser-generated hot electrons. FLYCHK determines time-dependent screened hydrogenic level populations by solving a coupled set of rate equations which include collisional excitation, deexcitation, ionization, and recombination as well as radiative emission, recombination, electron capture and autoionization processes. These level populations are used to determine the $K_{\alpha}$ signal as a function of time. As shown in figure 5 for the case of $10^{19}$ $\mathrm{W} / \mathrm{cm}^{2}$, the time duration (10-20 ps) of the modeled $\mathrm{K}_{\alpha}$ signal close to that measured by 
the experiment (15.9 ps). For the lower laser intensities, the agreement between the modeled $\mathrm{K}_{\alpha}$ duration (2-10 ps) and the measurements (12-13 ps) is less satisfactory. Moreover, even when the model matches the decay of the $K_{\alpha}$ intensities, it does not reproduce the measured rise. Unlike earlier studies at lower laser intensities $\left(10^{14}-10^{17}\right.$ $\left.\mathrm{W} / \mathrm{cm}^{2}\right)^{23,43}$ where the measured $\mathrm{K}_{\alpha}$ signal (10-15 ps) cannot be coincident in our experiment.

The $\mathrm{K}_{\alpha}$ signal seems to start before the peak in the laser pulse. Our interpretation is an initial population of hot electrons generates $\mathrm{K}_{\alpha}$ photons via inner-shell processes and created secondary " $\mathrm{K}_{\alpha}$ electron" via ionization of valence shell electrons (i.e., electrons with sufficient energy to produce further inner-shell ionizations). Since the rate coefficient for the valance-shell ionization is several hundred times that of inner-shell ionization, only a small fraction of these secondary electrons need to have sufficient energy to produce $\mathrm{K}_{\alpha}$ emission in order to overwhelm the direct $\mathrm{K}_{\alpha}$ production by the primary hot electrons. In this scenario, the risetime (and indeed the majority of the $\mathrm{K}_{\alpha}$ production) is attributable to these secondary $\mathrm{K}_{\alpha}$ electrons. Future measurements will help resolve the details of this measurement.

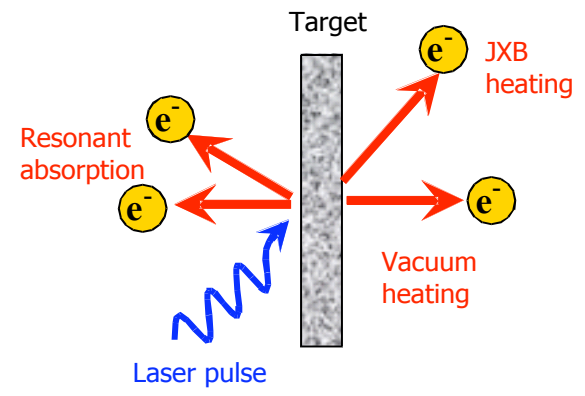

Figure 1 Illustration of the spatial features of hot electrons generated by three absorption mechanisms.

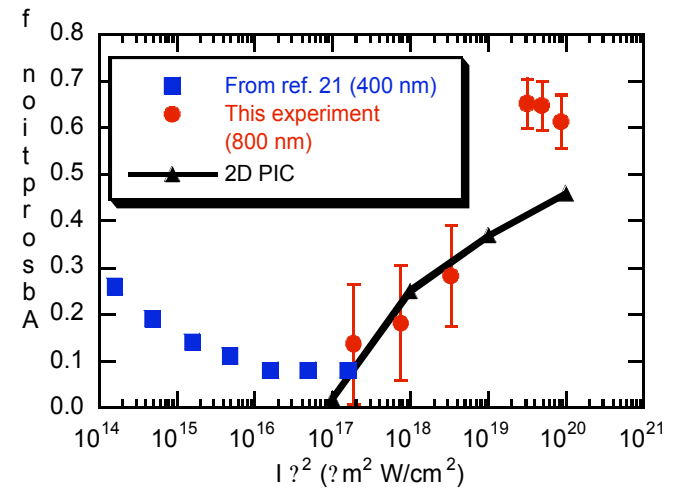

Figure 2a

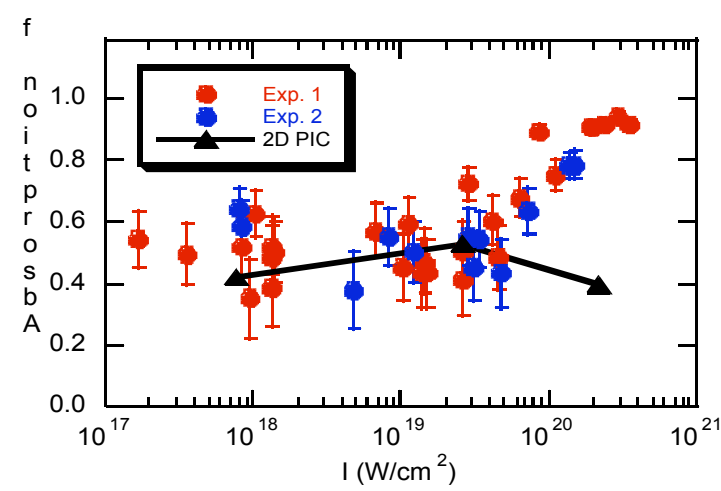

Figure $2 b$

Figure 2 Absorption fraction of the laser energy as a function of laser intensity at an incident angle of $6^{\circ}$ (a) and $45^{\circ}$ (b). Each point represents the result of a single laser shot. The error bars are standard deviations in instrumental calibration. The targets were Al foils with thickness 1.5-100 $\mu \mathrm{m}$ and 400- $\mu \mathrm{m}$-thick Si plates. Our measurements did not show significant dependence of absorption on these target thickness and materials. Therefore, the plots include data from various targets. 


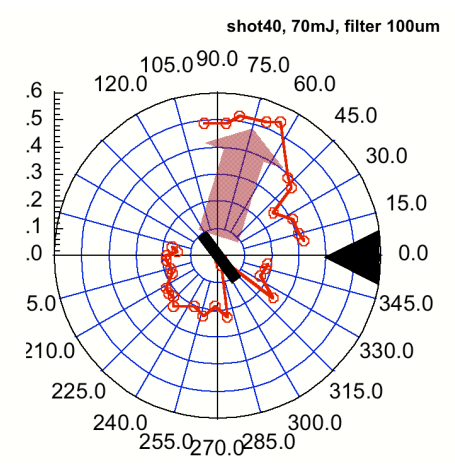

Figure 3a

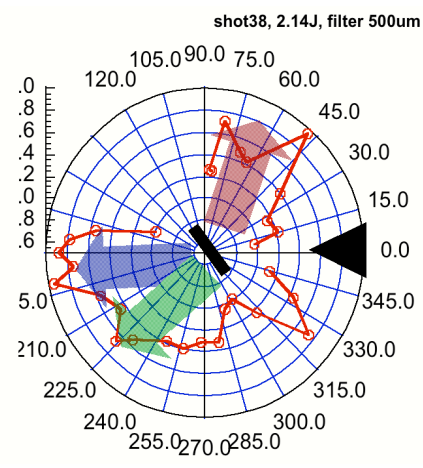

Figure $3 b$

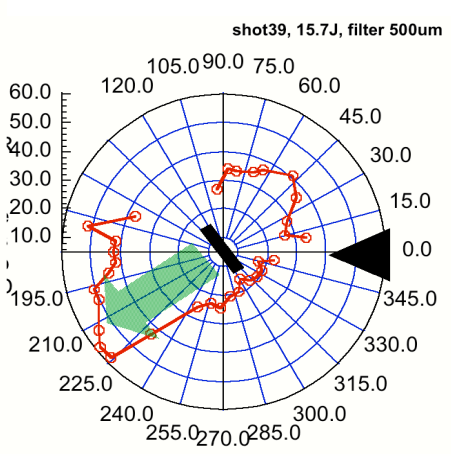

Figure $3 \mathrm{c}$

Figure 3 Angular distributions of hot electrons measured by dosimeters at three intensities. The targets were $10-\mu \mathrm{m}$-thick Al foils. From left to right: Figure $3 \mathrm{a}=\mathrm{I}=8 \times 10^{17} \mathrm{~W} / \mathrm{cm}^{2}$ (with $100-\mathrm{m}$-thick Al filters), Figure $3 \mathrm{~b}=2 \times 10^{19} \mathrm{~W} / \mathrm{cm}^{2}$ (with 500- m-thick Al filters), and Figure $3 \mathrm{c}=2 \times 10^{20} \mathrm{~W} / \mathrm{cm}^{2}$ (with 500 - mthick $\mathrm{Al}$ filters). The laser direction and the target orientation are indicated by the arrow and the black line at the center, respectively. The red, blue, and green arrows represent the hot electron directions for resonance absorption, $\overline{\boldsymbol{J}} \times \overline{\boldsymbol{B}}$ heating, and vacuum heating, respectively.

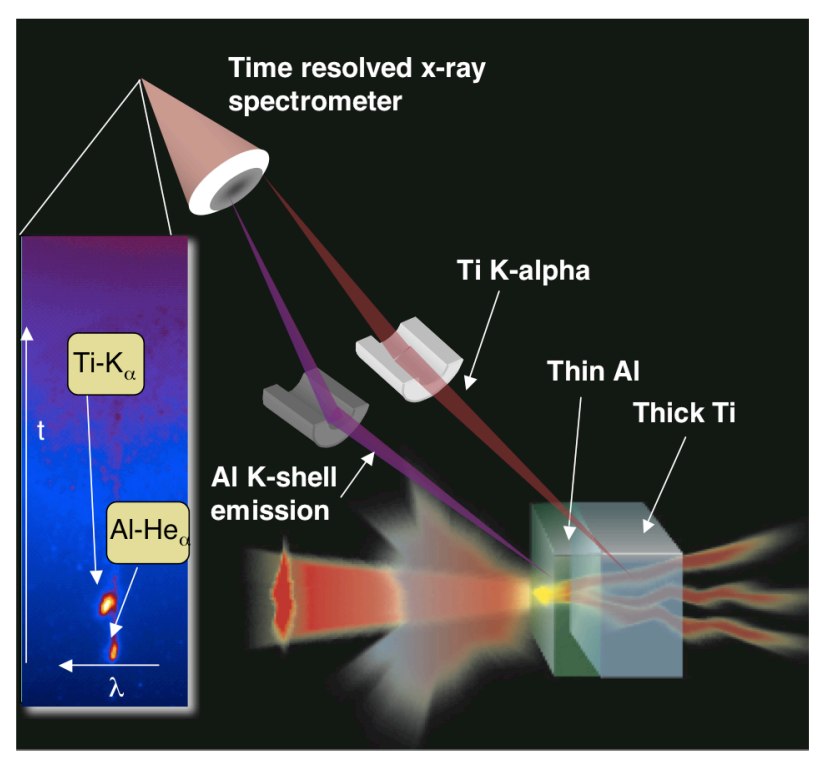

Figure 4: Experimental layout for the time-resolved $\mathrm{K}_{\alpha}$ experiment. The inset shows typical data from a shot. The spatial separation between the $\mathrm{Ti} \mathrm{K}_{\alpha}$ and $\mathrm{Al} \mathrm{He}{ }_{\alpha}$ results from the transit time difference of the xrays off the two crystals. 


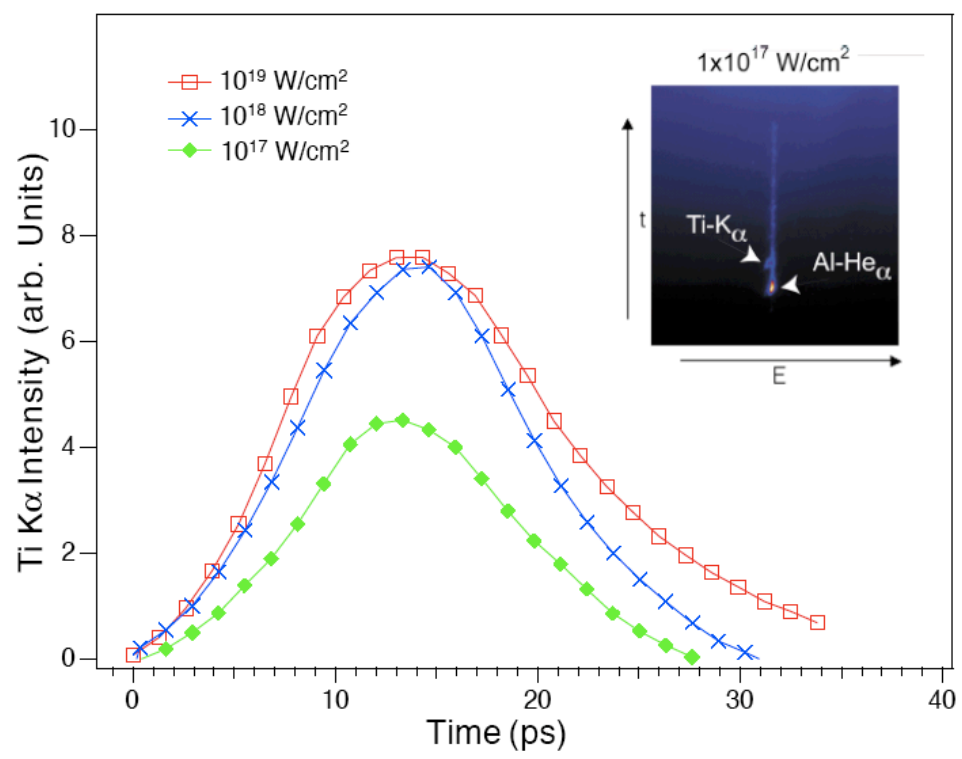

Figure 5: $\mathrm{Ti} \mathrm{K}_{\alpha}$ intensity versus time at three laser intensities. The full width at half maximum is $15.9 \mathrm{ps,}$ $13.2 \mathrm{ps}$, and $12.3 \mathrm{ps}$ measured at laser intensities of $10^{19}, 10^{18}$, and $10^{17} \mathrm{~W} / \mathrm{cm}^{2}$, respectively. The inset shows a streak image from the TREX streak camera at $10^{17} \mathrm{~W} / \mathrm{cm}^{2}$.

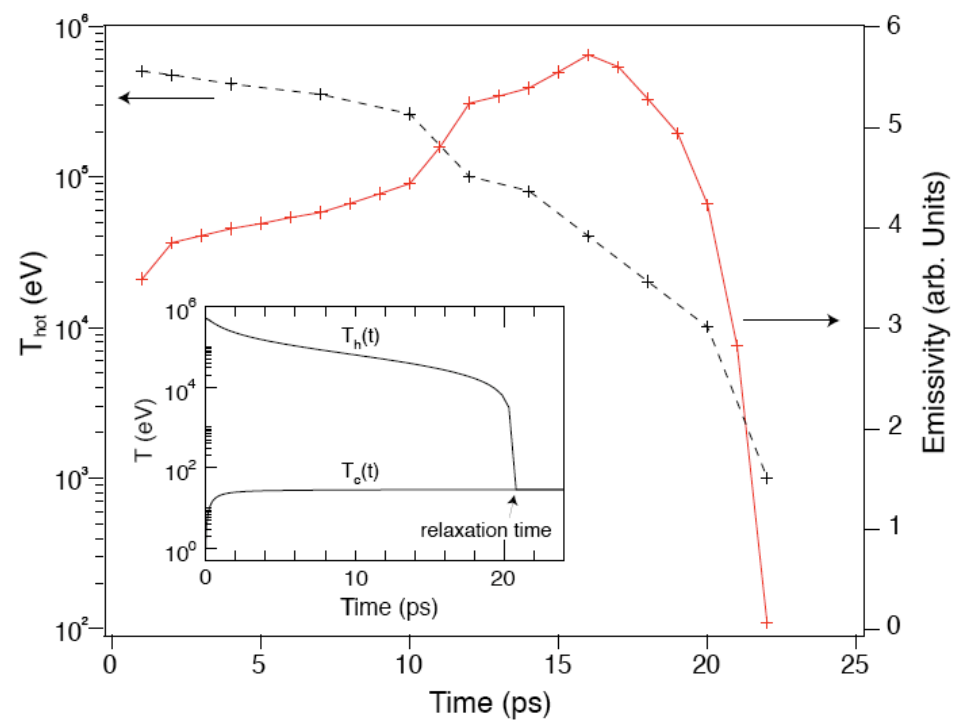

Figure 6: Calculated total $\mathrm{K}_{\alpha}$ emissivity (solid line) as a function of time. The hot electron relaxation used in this calculation (dashed line) was predicted by the collisional model shown in the inset assuming solid density $\mathrm{Ti}^{+4}$ plasma with $\mathrm{n}_{\mathrm{e} \text {-hot }}=10^{21} \mathrm{~W} / \mathrm{cm}^{2}$. evolution of the hot and cold electron temperature

\section{Summary}

These experiments have shown great promise for determining the characteristics of absorption and energy partition during solid target heating with high intensity short pulse lasers. The data and modeling will be used to design experiments for Omega-EP and NIF-ARC. We plan to extend these experiments to further quantify the energy transfer mechanisms for the relativistic electrons. These efforts will focus on understanding the effects of the damping rate on the $\mathrm{K}_{\alpha}$ time history and proton 
generation. Both efforts will be pursued through programmatic funding and future LDRD proposals.

\section{Acknowledgements}

We thank the laser operation team at Jupiter laser facility at LLNL. We would also like to acknowledge Dr. P. Patel, Dwight Price, Dr. Peter Beiersdorfer, for valuable discussions. Also we thank A. Niles, Jim Hunter, and Roger Van Maren for extensive technical support. This work was performed under the auspices of U.S. department of energy by LLNL under contract number W-7405-eng-48.

\section{References}

${ }^{1}$ ) R. Kodama, et. al., "Fast heating of ultrahigh-density plasma as a step towards laser fusion ignition". Nature, vol.412, no.6849, 23 Aug. 2001,pp.798-802

2 ) $\mathrm{Yu}$ Wei, et. al., "Ponderomotive acceleration of electrons at the focus of high intensity lasers". Phys. Rev. E., vol.61, no.3, March 2000, pp.R2220-3.

${ }^{3}$ ) L. Gremillet, et. al.,." Time-resolved observation of ultrahigh intensity laser-produced electron jets propagating through transparent solid targets. Physical Review Letters, vol.83, no.24, 13 Dec. 1999, pp.5015-18

$\left.{ }^{4}\right)$ M. Tabak, et. al., Phys. Plasmas, 1, 1626 (1994).

5 ) Y. Hironaka, et. al., "Enhancement of hard X-ray emission from a copper target by multiple shots of femtosecond laser pulses". Applied Physics Letters, vol.74, no.12, 22 March 1999, pp.1645-7

${ }^{6}$ Wilks, S. C. \& Kruer, W. L. Absorption of ultrashort, ultra-intense laser light by solids and overdense plasmas. IEEE J. Quant. Electron. 33, 1954-1968 (1997).

${ }^{7}$ Santala, M. I. K. et al. Absorption of ultra-intense laser-pulses. Phys. Rev. Lett. 84, 1459-1462 (2000).

${ }^{8}$ Zhang, J. et al. Emission direction of fast electrons in high-intensity laser interactions with solids. High Energy Density Phys. 1, 61-65 (2005).

${ }^{9}$ Price, D. F. et al. Absorption of ultrashort laser pulses by solid targets heated rapidly to temperatures 1 1000 eV. Phys. Rev. Lett. 75, 252-255 (1995).

${ }^{10}$ Gumbrell, E. T. et al. Picosecond optical probing of ultrafast energy transport in short pulse laser solid target interaction experiments. Phys. Plasmas 5, 3714-3721 (1998).

${ }^{11} \mathrm{Li}, \mathrm{Y}$. T. et al. High-energy electrons produced in subpicosecond laser-plasma interactions from subrelativistic laser intensities to relativistic intensities. Phys. Rev. E 69, 036405 (2004).

${ }^{12}$ Bilski, P., Olko, P., Burgkhardt, B. \& Piesch, E. Ultra-thin LiF:Mg,Cu,P detectors for beta dosimetry. Radiation Measurements 24, 439-443 (1995).

${ }^{13}$ Price, D. F. et al. Absorption of ultrashort laser pulses by solid targets heated rapidly to temperatures 11000 eV. Phys. Rev. Lett. 75, 252-255 (1995).

${ }^{14}$ Langdon, A. B. \& Lasinski, B. F. in Methods in Computational Physics (eds Killen, J., Alder, R., Fernbach, S. \& Rotenberg, M.) 327-366 (Academic Press, New York, 1976).

${ }^{15}$ Brunel, F. Not-so-resonant, resonant absorption. Phys. Rev. Lett. 59, $52-55$ (1987).

${ }^{16}$ Attwood, D. T., Sweeney, D. W., Auerbach, J. M. \& Lee, P. H. Y. Interferometric confirmation of radiation-pressure effects in laser-plasma interactions. Phys. Rev. Lett. 40, 184-186 (1978).

${ }^{17} \mathrm{Liu}, \mathrm{X}$., \& Umstadter, D. Competition between ponderomotive and thermal forces in short-scale-length laser plasma. Phys. Rev. Lett. 69, 1935-1938 (1992).

${ }^{18}$ Hansen, S. B. et al. Temperature determination using $\mathrm{K}$ spectra from M-shell Ti ions. Phys. Rev. E 72, 036408 (2005).

${ }^{19}$ Hegelich, B. M. et al. Laser acceleration of quasi-monoenergetic MeV ion beams. Nature 439, 441-444 (2006). 
${ }^{20}$ Schwoerer, H. et al. Laser-plasma acceleration of quasi-monoenergetic protons from microstructured targets. Nature 439, 445-448 (2006).

${ }^{21}$ Remington, B. A., Arnet, D., Drake, R. P. \& Takabe, H. Modeling astrophysical phenomena in the laboratory with intense lasers. Science 284, 1488-1493 (1999).

${ }^{22}$ S. P. Hatchett, et al., Phys. Plasmas, 7, 2076 (2000).

${ }^{23}$ K. B. Wharton, et al., Phys. Rev. Lett., 81, 822 (1998).

${ }^{24}$ R. A. Snavely, et al., Phys. Rev. Lett., 85, 2945 (2000).

${ }^{25}$ Ch. Reich, et al., Phys. Rev. E, 68,056408 (2003).

${ }^{26}$ D. Riley, et al., Phys. Rev. E, 71,016406 (2005).

${ }^{27}$ H. Chen, et al., Phys. Rev. Lett. 70, 3431 (1993).

${ }^{28}$ R. Stephens, et al., Phys. Rev. E, 66, 066414 (2004).

${ }^{29}$ J. Dunn, et al., Opt. Lett., 24, 101 - 103 (1999).

${ }^{30}$ J. Dunn, et al., in AIP Conf. Proc. vol. 369,"Laser Interaction and Related Plasma

Phenomena", ed. S. Nakai and G.H. Miley, pp. 652 - 659 (American Institute of Physics Press: Woodbury, New York) (1996)

${ }^{31}$ Liu, C. S. and M. N. Rosenbluth, Phys. Fluids, \$bf\{19\}\$, 967 (1976); L. Veisz, W. Theobald, T., Feurer, H. Schwoerer, I. Uschmann, O. Renner, and R. Sauerbrey, Phys. Plasma., 11, 3311 (2004).

${ }^{32}$ R. Shepherd, et al., Rev.Sci.Inst., 75, 3765 (2004).

${ }^{33}$ H. Chen, et al., Rev. Sci. Inst., 74, 1551, (2003).

${ }^{34}$ S. B. Hansen, et al., Phys. Rev. E, 72, 036408, (2005).

${ }^{35}$ Y. Ping, et al., submitted (2006).

${ }^{36}$ D. F. Cai, et al., Phys. Rev. E, 70, 066410, (2004).

${ }^{37}$ S. Bastiani, et al., Phys. Rev. E, 56, 7179, (1997).

${ }^{38}$ H. Ruhl, et al., Phys. Rev. Lett., 82, 743 (1999).

${ }^{39}$ Y. T. Li, et al., Phys. Rev. Lett., 96, 165003 (2006).

${ }^{40}$ D. F. Cai, et al., Phys. Plasmas, 10, 3265 (2003).

${ }^{41}$ Y. Sentoku, et al., Phys. Plasmas, 6, 2855 (1999).

${ }^{42}$ J. Scofield, Phys. Rev. A, 9, 1041, (1974).

${ }^{43}$ F. Pisani, et al., Phys. Rev. E, 62, R5927 (2000).

${ }^{44}$ P. Mora Phys. Rev. Lett., 90,185002 (2003).

${ }^{45}$ H-K., Chung, et al., High Energy Density Physics, 1, 3 (2005). 